\title{
Adherencia al uso de audífonos en adultos mayores con hipoacusia: Un ensayo clínico aleatorizado para evaluar un programa de rehabilitación auditiva
}

\section{Adherence to hearing aid use in older adults with hearing loss: A randomized clinical trial to evaluate a hearing rehabilitation program}

\author{
Felipe Cardemil M. ${ }^{1,2}$, Lorena Aguayo G. ${ }^{1}$, Eduardo Fuentes L. ${ }^{3}$, Daniel Muñoz S. ${ }^{1}$, \\ Tamara Barría E. ${ }^{1}$, Adrian Fuente C. ${ }^{4}$, Maritza Rahal E. ${ }^{1}$, Bevan Yueh. ${ }^{5}$, Graciela Rojas $C .{ }^{6}$
}

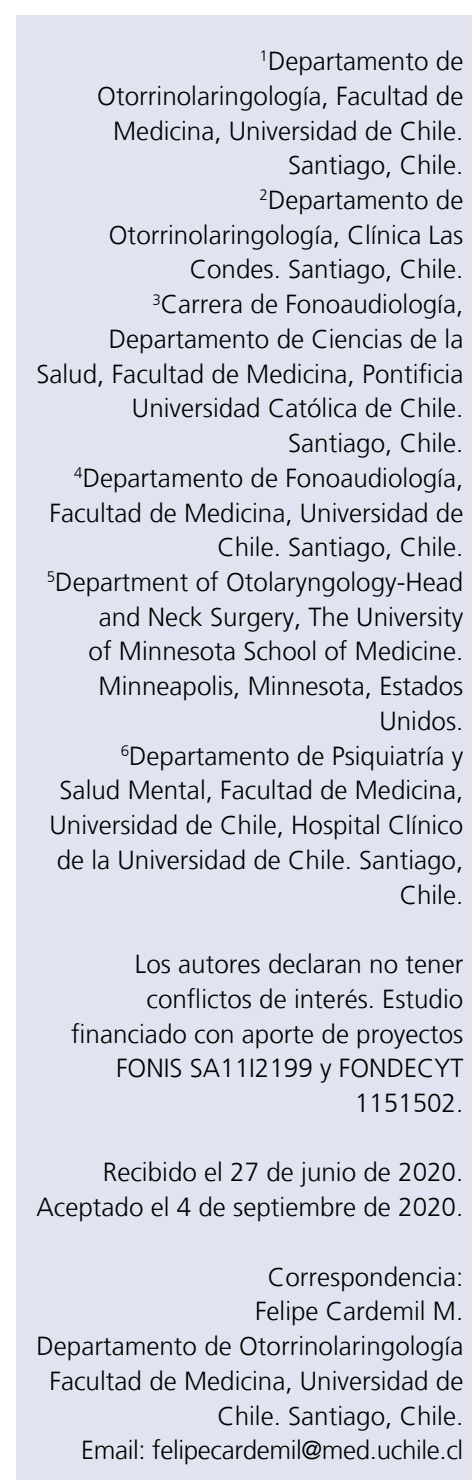

\section{Resumen}

Introducción: La presbiacusia es una condición muy prevalente. Habitualmente se implementan audífonos como tratamiento, pero un problema es la baja adherencia exhibida que puede rondar un $50 \%$. Objetivo: Evaluar si el programa de rehabilitación auditiva Active Communication Education (ACE) mejora la adherencia al uso del audífono. Material y Método: Se realizó un ensayo clínico aleatorizado controlado para evaluar la efectividad del programa ACE sumado a un refuerzo teleeducativo para mejorar la adherencia al uso del audífono. El proyecto fue aprobado por comité de ética y cada participante firmó consentimiento informado. La adherencia se evaluó por medio de la escala CIRUA y Dr. Yueh. Cada participante fue seguido en su domicilio en controles cada 3 meses hasta 1 año posterior a la intervención por encuestadores entrenados. Resultados: Se incluyeron 202 participantes, 101 en cada rama. La mediana de edad fue de 78 años y el 59,9\% de la muestra fue de sexo femenino. El PTP promedio óseo fue de $48,59 \mathrm{~dB}$ y el aéreo fue de $55 \mathrm{~dB}$. El grupo control tuvo adherencia de $65,91 \%$ y $62,30 \%$. El grupo intervencional tuvo una adherencia de $78,41 \%$ y $72,28 \%$. Hubo diferencias significativas en el porcentaje de adherencia entre grupo control y experimental según CIRUA $(\mathrm{p}=0,027)$ y Yueh $(\mathrm{p}=0,011)$. Conclusión: El programa de rehabilitación auditiva ACE es eficaz para mejorar la adherencia al uso del audífono en pacientes adultos mayores con hipoacusia. Este estudio representa uno de los mayores esfuerzos publicados para evaluar la efectividad de los programas de rehabilitación auditiva en adultos mayores con hipoacusia.

Palabras clave: Hipoacusia, adulto mayor, audífono, adherencia, rehabilitación.

\section{Abstract}

Introduction: Hearing loss associated with older adults is a very prevalent condition. Hearing aids are usually implemented as treatment, but there is low adherence exhibited, which can be around 50\%. Aim: To assess whether the Active Communication Education (ACE) hearing rehabilitation program improves adherence to hearing aid use. Material and Method: A randomized controlled clinical trial was carried out to evaluate the effectiveness of the ACE program added to a tele-educational reinforcement to improve adherence to hearing aid use. The project was approved by the ethics committee of the institution and each participant signed an informed consent. Adherence was evaluated using the CIRUA scale and Dr. Yueh scale. Each participant was followed at home in controls every 3 months up to 1 year after the intervention by trained interviewers. Results: 202 participants were included, 101 in each branch. The median age was 78 years and 59.9\% of the sample was female. The average bone PTA was $48.59 \mathrm{~dB}$ and air PTA was $55 \mathrm{~dB}$. Adherence 
in the control group was $65.91 \%$ and $62.30 \%$. In the interventional group, adherence was $78.41 \%$ and $72.28 \%$. There were significant differences in the percentage of adherence between the control and experimental groups according to CIRUA $(p=0.027)$ and Yueh $(p=0.011)$. Conclusion: The ACE auditory rehabilitation program improves adherence to hearing aid use in older adults with hearing loss. This study represents one of the largest published efforts to evaluate the effectiveness of auditory rehabilitation programs in older adults with hearing loss.

Keywords: Hearing loss, elderly people, hearing aids, adherence, rehabilitation.

\section{Introducción}

El envejecimiento poblacional es una tendencia epidemiológica global. En concordancia con esto, la hipoacusia asociada a los adultos mayores cada vez se convierte en una condición más prevalente ${ }^{1-3}$. La hipoacusia tiene impacto en la comunicación y la capacidad funcional, y está fuertemente asociada con la disminución en la calidad de vida, el deterioro cognitivo y alteración del ánimo ${ }^{4}$. A menudo es subestimada por ser un problema de lenta progresión o debido a la creencia de que la pérdida de audición es una parte normal de envejecimiento ${ }^{5}$. La pérdida de audición de $25 \mathrm{~dB}$ o más afecta a alrededor del $37 \%$ de los adultos de 61 a 70 años de edad, $60 \%$ de los adultos de 71 a 80 años de edad, y más del $80 \%$ de los adultos mayores de 85 años. De acuerdo a cifras de la Organización Panamericana de Salud (OPS), esta cifra fluctúa entre un 30\% en mayores de 65 años hasta $60 \%$ en mayores de 85 años $^{4}$. En adultos mayores chilenos, la prevalencia se estima en un $32,7 \%$, de acuerdo a datos de la Encuesta Nacional de Salud 2010 7 . Desde julio del año 2007, se incluye dentro de las Garantías Explícitas en Salud (GES) la entrega de audífonos a personas de 65 años y más con hipoacusia bilateral que lo requieran. Una de las razones por las cuales se diseñó esta política es porque existe información que apoya el que la hipoacusia genera costos importantes para un país, debido a disminución de la productividad, educación especial y atención en salud ${ }^{8}$.

Durante el tratamiento puede haber resistencia del paciente o la imposibilidad para implementar soluciones para la hipoacusia. El costo y el estigma social son factores importantes en el diagnóstico y tratamiento de la pérdida auditiva ${ }^{9-11}$. La principal motivación para el uso de audífonos, es la confianza del paciente en que la comunicación va a mejorar. Se ha observado que el uso de audífonos no se asocia con la edad, nivel de educación, el deterioro funcional o el uso de medicamentos. El asesoramiento debe ser proporcionado a los pacientes con pérdida de audición, porque las percepciones y expectativas del paciente son los factores más importantes en la adquisición de audífonos y su adherencia ${ }^{11-14}$.

La adherencia al uso de audífonos por parte de los pacientes hipoacúsicos sigue siendo un problema importante. En Chile se ha estimado en la Encuesta Nacional de Salud de 2003 una adherencia de 53\% en el sistema público ${ }^{4}$. Dentro de las causas de baja adherencia se describen factores asociados al paciente, tales como la predisposición al uso de audífonos, la autopercepción de la hipoacusia, las expectativas de mejoría, y otros. También se encuentran factores audiológicos y del sistema de salud que repercuten en disminuir la adherencia, como el seguimiento y rehabilitación auditiva. En base a esto, en 2014 se evalúo en Chile la utilidad de un protocolo de consejería estandarizado basado en el programa "Active Communication Education" (ACE) como intervención en pacientes con hipoacusia usuarios de audífonos para mejorar la adherencia a su uso ${ }^{15,16}$. Se observó que existían diferencias significativas respecto al tiempo de uso y adherencia al uso de audífonos al utilizar esta estrategia de consejería y educación basada en resolución de problemas, en un seguimiento a 6 meses.

\section{Objetivo}

El objetivo del presente estudio fue evaluar si el programa ACE sumado a un programa teleeducativo de monitorización mejoraba la adherencia al uso del audífono en un seguimiento a largo plazo. 


\section{Material y Método}

Se realizó un ensayo clínico aleatorizado controlado para el que se reclutaron participantes adultos mayores usuarios de audífonos beneficiarios del programa GES "Hipoacusia bilateral en mayores de 65 años que requieran audífonos" que acudieron a recibir atención en salud en el Servicio de Otorrinolaringología del Hospital Barros Luco Trudeau entre junio del año 2012 hasta diciembre del año 2014. Se realizó aleatorización en bloques permutados a través de un programa computacional. El protocolo del estudio fue registrado en el repositorio de protocolos de investigación ClinicalTrials.gov (identificador NCT02264314), fue aprobado por el Comité de Ética del Servicio de Salud Metropolitano Sur y de la Facultad de Medicina de la Universidad de Chile, y cada paciente firmó un consentimiento informado al ser enrolado en el estudio. Se consideraron las variables edad, sexo, promedio tonal puro entre las frecuencias $500 \mathrm{~Hz}$ a $4000 \mathrm{~Hz}$ (PTP) aéreo y óseo, discriminación de la palabra, tipo de hipoacusia y forma de la curva auditiva, escolaridad, uso previo de audífono, marca y modelo de audífono recibido, comorbilidades, y predisposición al uso de audífono.

Se aleatorizó en 2 ramas: una rama activa y una rama control. La intervención consistió en la aplicación de un programa de rehabilitación auditiva en grupos semiestructurados basado en el programa ACE de 4 sesiones de duración realizadas por un fonoaudiólogo con entrenamiento formal en la aplicación del programa, de una hora y media de duración, semanales. Posterior a la intervención del fonoaudiólogo se realizó una monitorización telefónica que consistía en que una profesional de la salud con entrenamiento formal en monitorización de ensayos clínicos, llamaba por teléfono a los participantes dos veces al mes durante los primeros tres meses posterior realización del programa de entrenamiento. Para la primera etapa del programa de rehabilitación auditiva basado en el ACE, que es un programa basado en solución de problemas, se adaptó el programa original que tiene 5 sesiones a un programa de 4 sesiones en grupos de 4 o 5 participantes, cada uno con un acompañante. Los módulos específicos que se abordan durante las sesiones del programa dependían de las necesidades comunicativas que eran identificadas por el grupo de participantes durante la primera sesión, por lo que los contenidos variaban dependiendo de las dificultades de comunicación específicas descritas por los participantes. En la monitorización telefónica se explicó el anexo 9 ("Instructivo para usuarios: Expectativas de audición con audífonos") y el anexo 12 ("Consejos útiles para un mejor uso de su audífono") que estipulaba la guía GES. La rama control (pasiva) consistió en la asistencia habitual que recibían estos usuarios luego de la entrega del audífono en el sistema de atención público chileno, que corresponde a control en caso de presentar algún desperfecto en el audífono, sin la aplicación de un programa de entrenamiento de rehabilitación auditiva formal. Debido a la característica de la intervención, no fue posible enmascarar a los participantes del estudio ni al personal del estudio. Al término del estudio, y según lo estipulado en el comité de ética, se les ofreció a los participantes de la rama pasiva ser parte de la intervención que recibió la rama activa.

El seguimiento de los pacientes se realizó cada 3 meses (totalizando cuatro controles: a los 3, 6, 9 y 12 meses posterior a la realización de la intervención) por encuestadores específicamente contratados y entrenados para los fines de este estudio, los que acudieron a la casa de cada participante hasta en tres oportunidades por cada control (en caso que no los encontraran en su domicilio en alguna de las visitas).

Se realizó análisis por intención de tratar. El desenlace primario a evaluar fue la adherencia al uso del audífono aplicándose el International Outcome Inventory for Hearing Aids (IOI-HA) o Cuestionario Internacional del Resultado de Uso de Audífonos (CIRUA), considerando la pregunta 1 de este cuestionario, y un cuestionario de adherencia proporcionado por el Dr. Bevan Yueh (Figura 1). Con éstos se estimó la adherencia en términos de uso general y tiempo de uso, además de variables demográficas y auditivas habituales.

Para el cálculo de tamaño muestral, considerando $\alpha=5 \%$ y $\beta=20 \%$, y una frecuencia de adherencia al uso de audífonos reportada de $53 \%$, que se pretende aumentar a $75 \%$, fue de 83 usuarios por cada uno de los brazos. Para los fines de este estudio, se consideraron 
100 usuarios en cada grupo, asumiendo hasta un $20 \%$ de pérdida, por lo que el tamaño final fue de 200 usuarios. Para el análisis estadístico se utilizó el programa estadístico Stata 12. Las variables continuas fueron descritas mediante promedio (desviación estándar) o mediana (rango intercuartil -RIC-) según correspondiera, y las variables categóricas mediante frecuencias absolutas y relativas. Se utilizó la prueba de Shapiro Wilk para evaluar la distribución de las variables continuas, y en base a si seguían una distribución normal o no, las variables continuas fueron comparadas con la prueba $t$ de student o el test de Mann Whitney, según correspondiera. Para las variables categóricas la comparación entre grupos se realizó utilizando el test exacto de Fisher. Se utilizó como nivel de significación un $\mathrm{a}=0,05$.

\section{Resultados}

Fueron evaluados para potencial enrolamiento 264 participantes, de los cuales 48 no cumplían con los criterios de inclusión, 3 renunciaron a participar y 11 fueron excluidos por otras razones. La muestra final quedó conformada por 202 participantes, correspondiendo a 101 participantes en cada rama. La mediana de edad (RIC) de los participantes fue 78 años (72-82), sin diferencias entre las ramas $(\mathrm{p}=0,17)$. El 59,9\% de la muestra fue de sexo femenino, sin diferencia significativa entre las ramas del estudio $(\mathrm{p}=0,38)$. Tampoco hubo diferencia significativa en la distribución de escolaridad entre las ramas de estudio $(\mathrm{p}=0,23)$.

Respecto a variables audiológicas, el PTP promedio óseo fue de $48,59 \mathrm{~dB}(10,25)$, con un rango de 22,5 a $70 \mathrm{~dB}$. No hubo diferencias significativas en el nivel auditivo óseo según el grupo en estudio ( $p=0,1442)$. El PTP promedio aéreo fue de $55 \mathrm{~dB}(47,5-65 \mathrm{~dB})$. No hubo diferencias significativas en el nivel auditivo aéreo según el grupo en estudio $(\mathrm{p}=0,7622)$. La discriminación auditiva tuvo una mediana de porcentaje de $80 \%(64-92 \%)$. No hubo diferencias significativas en la discriminación auditiva al comparar los grupos en estudio $(\mathrm{p}=0,9012)$. La distribución según el tipo de hipoacusia fue sensorioneural en un $87,82 \%$ $(n=173)$ y mixta en un $12,18 \%(n=24)$. No se encontraron diferencias significativas en

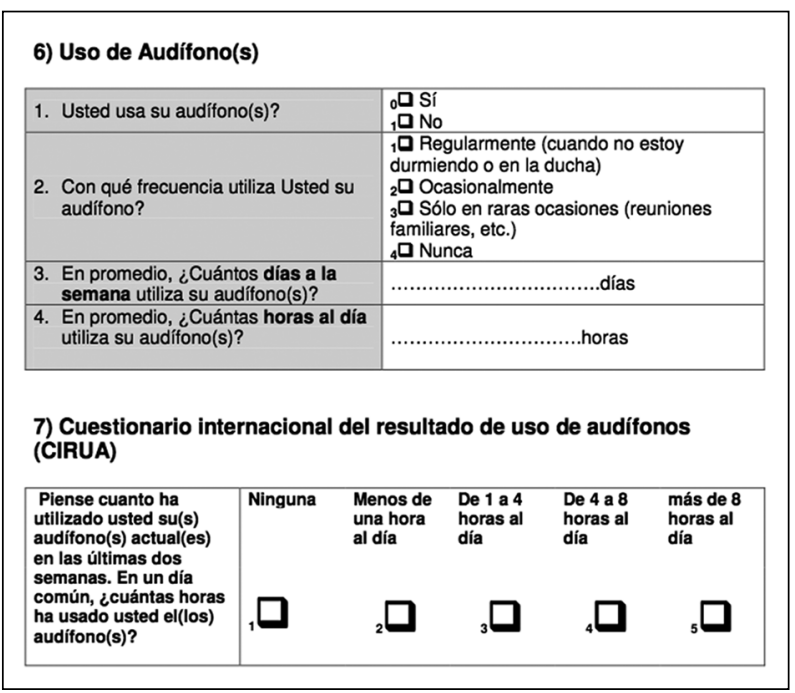

Figura 1. Instrumentos utilizados para evaluar adherencia al uso de audífono en la muestra seleccionada.

la distribución del tipo de hipoacusia según grupo en estudio $(p=0,514)$. La distribución según la forma de la curva audiométrica fue descendente en un $67,69 \%(n=132)$, plana en un $16,92 \%(\mathrm{n}=33)$ y plana-descendente en un $15,38 \%(n=30)$. No hubo diferencias significativas en la distribución según curva audiométrica al comparar según grupo en estudio $(\mathrm{p}=0,122)$. El uso previo de audífonos se observó en un $24,87 \%(n=47)$. No hubo diferencias en la distribución del uso previo de audífonos al comparar según grupo en estudio $(\mathrm{p}=0.401)$. La predisposición al uso de audífono se distribuyó en la muestra de la siguiente manera: muy mala $2,54 \%(\mathrm{n}=5)$, mala $0,51 \%(\mathrm{n}=1)$, regular $5,08 \%(\mathrm{n}=10)$, buena $37,56 \%(\mathrm{n}=74)$ y muy buena $54,31 \%$ $(\mathrm{n}=107)$. No hubo diferencias significativas en la distribución según predisposición al uso de audífono entre grupo activo y grupo control $(p=0,323)$. La adherencia al uso de audífonos, definida como el uso del aparato de al menos 8 horas diarias, se midió a través de la pregunta 1 del cuestionario CIRUA y de la pregunta 4 del cuestionario del Dr. Yueh, ambas considerando el seguimiento durante los 12 meses de participación. Según esto, se observó una adherencia general en la muestra de $72,28 \%(\mathrm{n}=146)$ y de $78,22 \%(\mathrm{n}=158)$ según CIRUA y Yueh, respectivamente. 
Al desglosar por grupo, se observó en el grupo control una adherencia de 65,91\% $(\mathrm{n}=$ $58)$ según CIRUA y de $62,30 \%(n=60)$ según Yueh. Por otro lado, en el grupo intervencional se observó una adherencia de 78,41\% $(\mathrm{n}=69)$ según CIRUA y de $72,28 \%(\mathrm{n}=73)$ según Yueh, siendo estas diferencias en el porcentaje de adherencia entre grupo control y experimental estadísticamente significativas según CIRUA ( $\mathrm{p}=0,027)$ (Figura 2$)$ y Yueh $(\mathrm{p}=0,011)$ (Figura 3$)$. En ninguna de las mediciones puntuales (tiempos de medición) la frecuencia de pérdida de datos fue superior al $7 \%$ de la muestra.

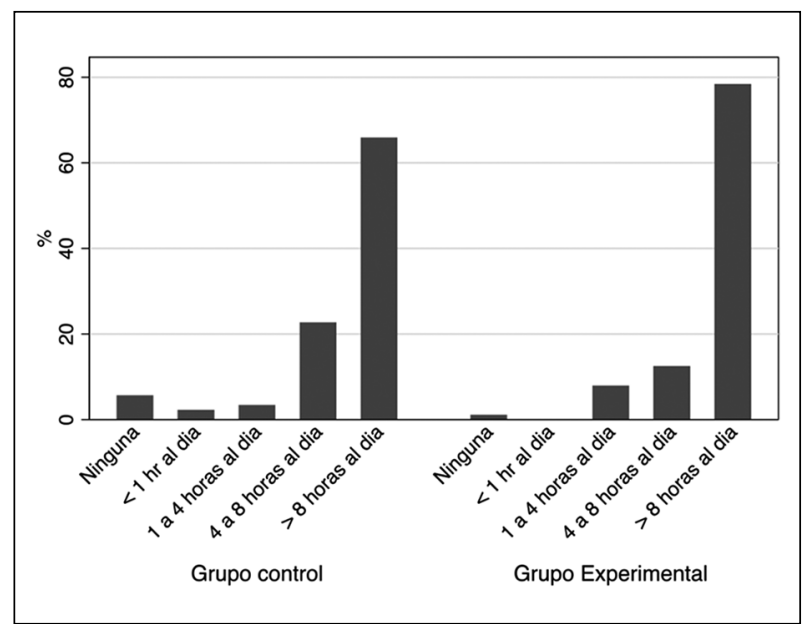

Figura 2. Distribución de los tiempos de uso de audífono a 12 meses según cuestionario CIRUA, separados por grupo intervencional y control. CIRUA: Cuestionario Internacional del Resultado de Uso de Audífonos

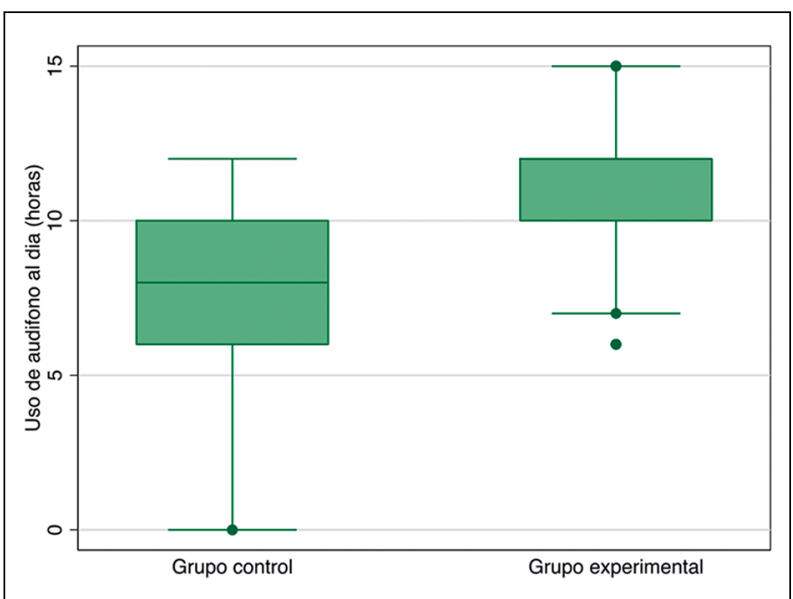

Figura 3. Distribución del tiempo de uso de audífono al día en un punto de corte a los 12 meses según cuestionario del Dr. Yueh, separados en grupo intervencional y grupo control.

\section{Discusión}

Habitualmente el tratamiento utilizado en el manejo de la hipoacusia del adulto mayor es la implementación con audífonos. Sin embargo, existe un porcentaje importante de pacientes que finalmente no lo utilizan, por lo que progresivamente se ha ido estudiando el rol que cumplen diferentes programas de rehabilitación auditiva en la mejora auditiva y la adherencia al uso del audífono en dichos pacientes. La evidencia actual sugiere que las intervenciones de rehabilitación, tanto individuales como grupales, generan resultados positivos, sin embargo, su disponibilidad en nuestro medio no siempre es la ideal. Para mejorar esta situación, se han propuesto cambios en el manejo de los pacientes con hipoacusia, mencionándose que la disponibilidad de la gama de intervenciones de rehabilitación debe ser mejorada, y los adultos mayores con discapacidad auditiva deben ser invitados a participar activamente en su rehabilitación ${ }^{17}$.

Los programas de rehabilitación auditiva grupal, a diferencia de los programas de entrenamiento auditivo individual, se basan en la entrega de orientación y estrategias comunicacionales para enfrentar mejor el déficit en la participación social. Distintos autores han mencionado la importancia de incorporar estrategias de comunicación y orientación como parte de un manejo integral de adultos con hipoacusia a través de un método eficiente, en tiempo y costo, en el contexto de rehabilitación grupal, donde es posible que los participantes compartan sus sentimientos, problemas y soluciones para enfrentar los problemas de comunicación ${ }^{1}$. Los programas de rehabilitación auditiva grupal usualmente incluyen la utilización de clases dirigidas en las cuales se incorpora información acerca de la audición, hipoacusia, lectura labial, estrategias comunicativas y dispositivos de asistencia personal. Por otra parte, algunos de estos programas también incluyen una práctica en la utilización de estrategias comunicacionales, el uso de técnicas de relajación y manejo de estrés, aspectos psicosociales, y la incorporación de la pareja. La mayoría de estos estudios ha demostrado beneficios de programas de rehabilitación auditiva grupal, en términos de reducción de la restricción en la participación social ${ }^{18,19}$. 
Particularmente en nuestra población, por medio de esta investigación, hemos observado que existen diferencias significativas respecto al tiempo de uso y adherencia al uso de audífonos al utilizar esta estrategia de consejería y educación basada en resolución de problemas en el contexto de la rehabilitación auditiva grupal, en los pacientes beneficiarios sometidos a la intervención. Se logró optimizar un programa de alta importancia social, educacional y de rehabilitación, generando un beneficio directo para los usuarios, a través de un programa estructurado de acuerdo a las necesidades particulares de cada grupo de pacientes intervenidos ${ }^{15,16}$. En forma importante, este ensayo clínico demuestra con evidencia de alto nivel que el impacto en la adherencia al uso del audífono aumenta significativamente con el uso de un programa de rehabilitación auditiva en adultos mayores chilenos, y que este efecto se mantiene hasta al menos 12 meses posterior a la intervención.

Utilizar una variedad de herramientas educativas para aumentar las oportunidades de aprendizaje de los pacientes permitiría un mejor resultado clínico en la rehabilitación auditiva. Al mejorar la adherencia terapéutica al uso de audífonos, se puede obtener un impacto directo en términos de mejoría de calidad de vida de los usuarios, e indirecto en términos de mejor utilización de un recurso que tiene un costo anual elevado para el país. La intervención implementada impresiona tener buenos resultados en términos de tiempo de uso del audífono, pudiendo ser de utilidad implementarla de manera reglada como parte de las políticas públicas y difundir su uso.

Dentro de las fortalezas de este estudio se encuentra el ser un ensayo clínico aleatorizado controlado con un sistema de aleatorización por bloques muy robusto, con un número de participantes elevado en comparación a otros estudios internacionales, con un período de seguimiento prolongado en comparación a las otras series publicadas, y con un método de seguimiento en el domicilio de los participantes y con encuestadores entrenados. Además de eso, incluye una innovación al considerar un refuerzo telefónico de la intervención, lo que consideramos hace más robusta a la intervención. Dentro de las debilidades del presente estudio podría considerarse haberse realizado en un centro único, y las pérdidas de datos, las que en todo caso no excedieron un 7\%. Por último, a pesar que la adherencia observada en el grupo intervenido fue elevada y significativamente superior a la del grupo control, la adherencia al grupo control fue superior a la estimada en el cálculo de tamaño muestral, por lo que el estudio pudiera no tener la potencia estadística para el cual fue diseñado. Sin embargo, es uno de los estudios que indagan en este tópico con mayor número de participantes publicado en la literatura internacional.

\section{Conclusión}

El programa de rehabilitación auditiva Active Communication Education impresiona ser eficaz para mejorar la adherencia al uso del audífono en pacientes adultos mayores con hipoacusia, presentándose como una herramienta útil en el manejo de estos pacientes a largo plazo. Los resultados de este ensayo clínico sugieren que la incorporación de programas de rehabilitación auditiva con un refuerzo teleeducativo en pacientes usuarios de audífonos podría resultar beneficiosa para aumentar la adherencia al uso del audífono. Este estudio representa uno de los mayores esfuerzos publicados en la literatura actual para indagar la efectividad de los programas de rehabilitación auditiva en la mejoría de la adherencia al uso de audífonos en adultos mayores con hipoacusia.

\section{Bibliografía}

1. Yueh B, Shapiro N, Maclean Ch, Shekelle Pg. Screening and management of adult hearing loss in primary care: scientific review. JAMA. 2003;289(15): 1976-1985.

2. Van Eyken E, Van Camp G, Van Laer L. The complexity of age-related hearing impairment: contributing environmental and genetic factors. Audio Neurootol. 2007;12(6):345-358.

3. Gates GA, Cooper JC JR, Kannel WB, Miller NJ. Hearing in the elderly: the Framinghan cohort, 19831985. Part 1. Basic audiometric tests results. Ear Hear. 1990;11(4):257-256.

4. Mulrow CD, Aguilar C, Endicott JE, Vélez R, Tuley MR, Charlip WS, et al. Association between hearing impairment and the quality of life of elderly individuals. J Am Geriatr Soc. 1990;38(1):45-50. 
5. Leonard R, Horowitz A. Hearing problems of and the need for hearing services by consumers of vision rehabilitation services. Journal of Visual Impairment and Blindness. 2004;98(3):168-172.

6. Patterson C. Prevention of hearing impairment and disability in the elderly. In: Canadian Task Force on the Periodic Health Examination. Canadian Guide to Clinical Preventive Health Care. 1994;954-63.

7. Ministerio de Salud, Chile, Encuesta Nacional de Salud año 2003. http://epi.minsal.cl/epi/html/ invest/ ENS/folletoENS.pdf (Revisión actualizada el 24 de agosto del año 2010).

8. Guía Clínica Hipoacusia bilateral en personas de 65 años y más que requieren uso de audífono. http:// www.redsalud.gov.cl/archivos/guiasges/ hipoacusia. pdf.

9. Garstecki DC, Erler SR. Hearing loss, control, and demographic factors influencing hearing aid use among older adults. J Speech Lang Hear Res. 1998;41(3):527-537.

10. Food and Drug Administrations Regulations: Hearing aid devices: professional and patient labeling and condition or sale. Audiology Update. 1993;12: 3-5.

11. Kochkin S, Marketrak Iv. What is the viable market for hearing aids? Hearing J. 1997;50(1):31-39.

12. Gussekloo J, De Bont Le, Von Faber M, et al. Auditory rehabilitation of older people from the general population-the Leiden 85-plus study. $\mathrm{Br} J \mathrm{Gen}$ Pract. 2003;53(492):536-40.
13. Pacal JT, Yueh B. Hearing deficits in the older patient: "I didn't notice anything". JAMA. 2012; 307(11):1185-1194.

14. Laplante-Lévesque A, Hickson L, Worrall L. Rehabilitation of older adults with hearing impairment: a critical review. J Aging Health. 2010;22(2):143-153.

15. Cardemil F, Barría T, Aguayo L, et al. Evaluación del programa "Active Communication Education" para rehabilitación auditiva en adultos mayores con hipoacusia usuarios de audífonos. Rev Otorrinolaringol Cir Cabeza Cuello. 2014;74(2):93100.

16. Hickson L, Worrall L, Scarinci N. A randomized controlled trial evaluating the active communication education program for older people with hearing impairment. Ear Hear. 2007;28:212-230.

17. Cardemil F, Aguayo L, Fuente A. Programas de rehabilitación auditiva en adultos mayores, ¿qué sabemos de su efectividad? Acta Otorrinolaringol Esp. 2014;65(4):249-257.

18. Collins M, Liu C, Taylor L, Souza PE, Yueh B. Hearing aid effectiveness after aural rehabilitation: Individual versus group trial results. JRRD. 2013; 50(4):585-598.

19. Hawkins DB. Effectiveness of counselling-based adult group aural rehabilitation programs: A systematic review of the evidence. J Am Acad Audiol. 2005;16(7):485-493. 